\title{
Molecular signatures of Alzheimer's disease and aging in the TOMM40-APOE-APOC1 locus
}

\author{
A.M. Kulminski*, L. Shu, Y. Loika, I. Culminskaya \\ Biodemography of Aging Research Unit, Social Science Research Institute, Duke University, Durham, USA \\ *e-mail: Alexander.Kulminski@duke.edu
}

Key words: Alzheimer's disease, aging, linkage disequilibrium, molecular signatures

Motivation and Aim: Enduring interest to the apolipoprotein E (APOE) region is driven by remarkably strong associations of variants from this region with Alzheimer's diseases (AD) and pleiotropic associations with multiple aging-related traits. The role of this region in pathogenesis of AD and aging remains, however, poorly understood. Elucidating genetic predisposition to aging-related traits characteristic for post-reproductive period is hampered by the uncertain role of evolution in establishing their molecular mechanisms. This uncertainty is inevitable source of natural-selection-free genetic heterogeneity in predisposition to AD and aging-related traits.

Methods and Algorithms: We examined linkage disequilibrium (LD) structures characterized by nine single nucleotide polymorphisms (SNPs) from TOMM40-APOE$A P O C 1$ locus, including rs429358 and rs7412 SNPs coding the APOE \&4 and $\varepsilon 2$ alleles, in 2,661 AD-affected and 16,079 AD-unaffected subjects and in 570 short-lived ( $<75$ years, SL) and 1,999 long-living ( $85+$ years, LL) subjects from four independent studies.

Results: The LD structures, being heterogeneous, are significantly different in subjects with and without $\mathrm{AD}, p<2 \times 10^{-4}$. The pattern of the significant difference represents molecular signature of AD comprised of SNPs from these genes. We identified 31 of 36 SNP pairs with pair-wise estimates of the LD difference between subjects with and without $\mathrm{AD}$ significant after Bonferroni correction, $p<1.3 \times 10^{-3}$. In contrast, differences in LD between SL and LL subjects attained only marginal Bonferroni-adjusted significance $\left(p=3.5 \times 10^{-3}\right)$ for one SNP pair (rs405509 [APOE] and rs439401 [intergenic]), nominal significance $\left(p<5 \times 10^{-2}\right)$ for three pairs and suggestive significance $\left(p<10^{-1}\right)$ for three more pairs. For all these seven SNP pairs, LD changed in the same directions in AD/ no-AD and LL/SL groups.

Conclusion: Significant and highly heterogeneous molecular signature of AD provides evidence on complex polygenetic predisposition to AD in the TOMM40-APOE-APOC1 locus. Significant differences in pair-wise LD in subjects with and without AD indicate SNPs, or their proxies, likely involved in AD pathogenesis. The same directions of the $\mathrm{LD}$ differences in $\mathrm{AD} /$ no-AD and LL/SL groups suggests heterogeneous, partly overlapping molecular mechanisms for $\mathrm{AD}$ and aging, defined as survival to old ages, in the $A P O E$ region.

Acknowledgements: Supported by Grants P01 AG043352 and R01AG047310 from the National Institute on Aging. 